\title{
Design of MULTI-Robot SySTEM For CleANING UP MARINE OIL SPILL
}

\author{
Emaad Mohamed H. Zahugi ${ }^{1}$, Mohamed M. Shanta ${ }^{2}$ and T. V. Prasad ${ }^{3}$ \\ ${ }^{1}$ Dept. of CSE, Lingaya's University, Faridabad, India \\ zahugi@yahoo.com \\ ${ }^{2}$ Dept. of CSE, Lingaya's University, Faridabad, India \\ mohshanta@yahoo.com \\ ${ }^{3}$ Dean (Computing Sciences), Visvodaya Technical Academy, Kavali, India \\ tvprasad2002@yahoo.com
}

\begin{abstract}
The researchers and oil companies are trying to take some precaution for the problem of oil spill in sea, river or on ground etc. A lot of work concerned by removing the oil from water, there were many advanced tools for this task This paper presents a multi-robot system that works on the surface of water to help cleaning up marine oil spills using a skimmer as a collecting tool, the aim of this multi-robot system is to surround the oil spill in certain position for fast and easy cleaning and prevent it from spreading wider.
\end{abstract}

\section{KEY WORDS}

Multi-Robot, Oil spill, wireless communication, robotics.

\section{INTRODUCTION}

Oil or "black gold" is still the largest source of power used by the industry sector. The demand for oil is increasing day-by-day and is substantiated by expanding submarine oil pipelines, distribution of oil and its derivatives by using tankers to carry it to many destinations. This leads to increased chances of oil leakage in the sea either by leakage from submarine oil pipelines or accidents with the tankers. In the past, such mis-happenings lead to major disasters of oil leakage in the high seas. Oil spills threaten the wildlife in the sea and hence, there is necessity for research on cleaning up oil spill quickly and efficiently has become very important issue by researchers and companies concerned. Last such disaster was the British Petroleum (BP) oil disaster in the Gulf of Mexico in USA during April 2010.

Recovering oil from water may cost billions of dollars and take too much time. It can last for weeks and months. Many researchers and designers are concerned with how to recover the oil from water starting with the process of detecting and monitoring oil spills [1-4]. Some researchers are concerned with the behavior of oil released from pipelines [5], numerical models and simulation programs for submarine oil spill $[6,7,8]$. Other researches were about removing the oil spill from the water with different technologies $[9,10]$ including but not limited to the following as shown in Figure 1:

DOI : 10.5121/ijait.2012.2404 
International Journal of Advanced Information Technology (IJAIT) Vol. 2, No.4, August 2012

a) bioremediation use of microorganisms or biological agents to break down or remove oil,

b) Controlled burning can effectively reduce the amount of oil in water, if done properly, but it can only be done in low wind and can cause air pollution,

c) Solidifying, Solidifiers are composed of dry hydrophobic polymers that both adsorb and absorb. They clean up oil spills by changing the physical state of spilled oil from liquid to a semi-solid or a rubber-like material that floats on water,

d) Skimming

e) Vacuum.

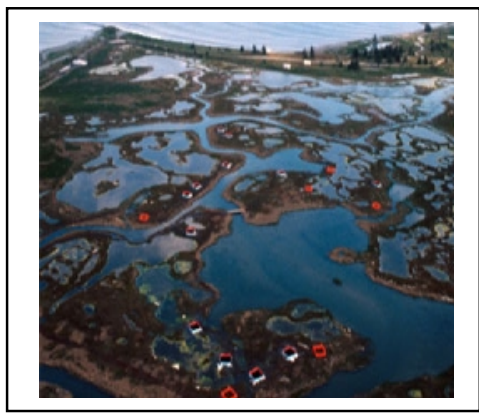

(a)

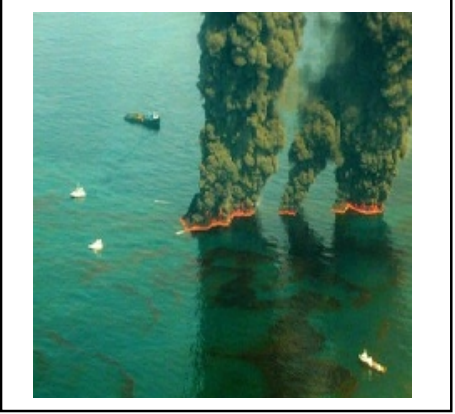

(b)

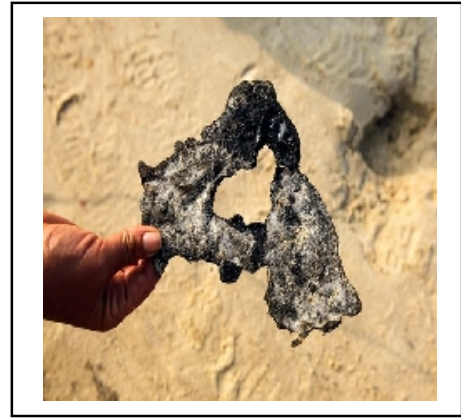

(c)

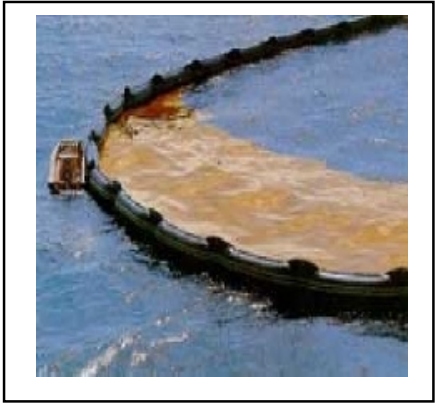

(d)

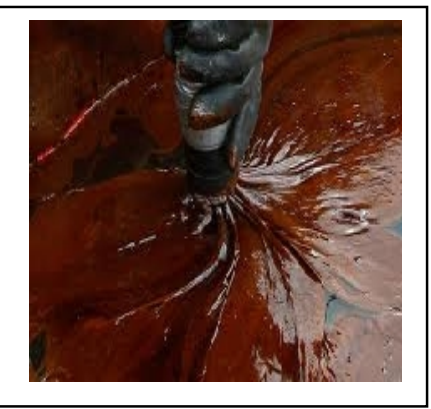

(e)

Figure 1. Methods of removing oil from water
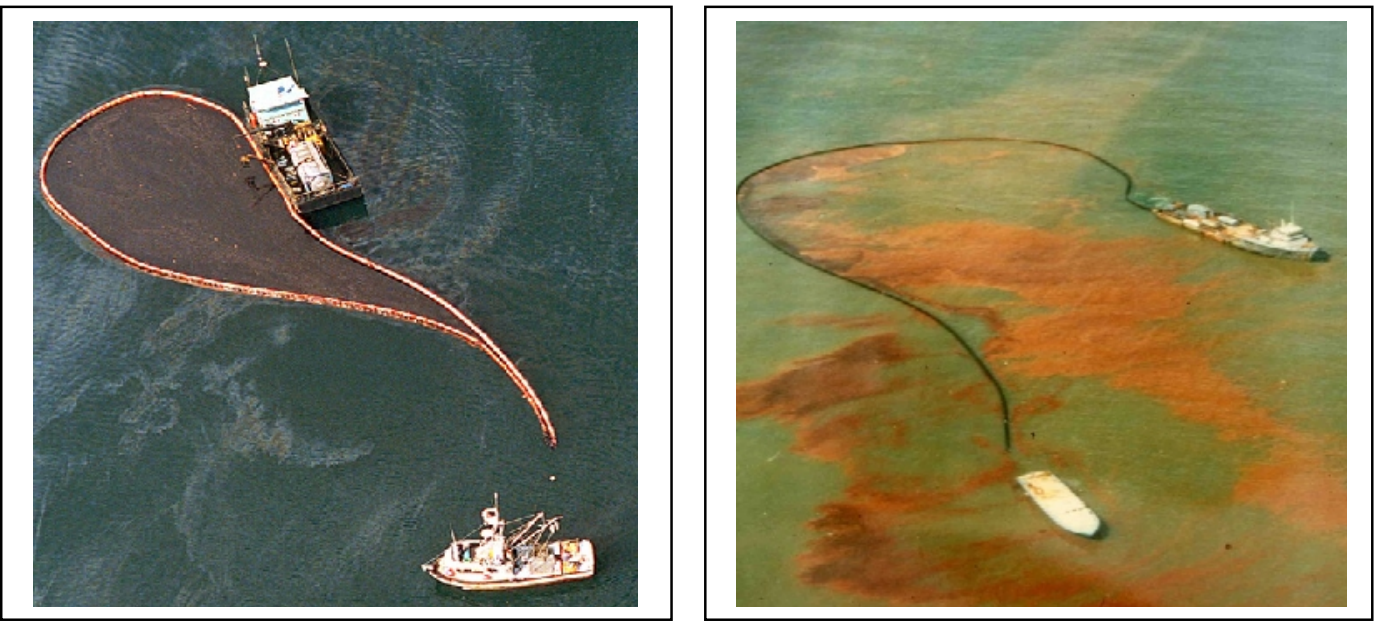

Figure 2.Two large barges collecting an oil spill in sea. 
Swarm robotics is a new research area, however its applications is still in its early stage and can be seen only inside labs. Swarm robotics applications includes but not limited to the following: (i) Exploring an open area like in boarders, (ii) Rescue tasks, in places that are dangerous for people to get in such as when there is fire, gas leakage, nuclear radiation or toxic substances, (iii) Agriculture foraging tasks, etc.

All the mentioned methods and technique concerned with removing the oil spill from water only but which is not an easy task as the spill usually gets stretched and spreads wider by passage of time. The only technology used to collect the oil spill is by using booms that are large floating barriers which round up the oil spill and then lift the oil off the water between two vessels as shown in Figure 2. This process takes much time and is expensive as vessels with large containers have to leave the place many times to dispose the recovered oil. In case the water is turbulent, the spill may get spread wider making it difficult to finish the process of cleaning up. Instead of using many large barges in the working area a swarm of sea robots equipped with a skimmers and booms can be used to collect the oil spill in one position and restrict its spread. Only vessels with containers can be there to suck the oil and store it. Also a swarm of sea robots can be dispatched to prevent an oil spill from moving towards seashore, coastline, harbor or any specific area and save the wild life more efficiently.

Researchers working with underwater robots aim to enable robots to perform many applications such as bio-mimetic swimming robots [11, 12, 13], military applications [14] education and research [15], also Unmanned Marine Vehicle is an important research area [16]. The proposed robot with modification can be used also in the search and rescue tasks in sea like when there is airplane crash in the sea.

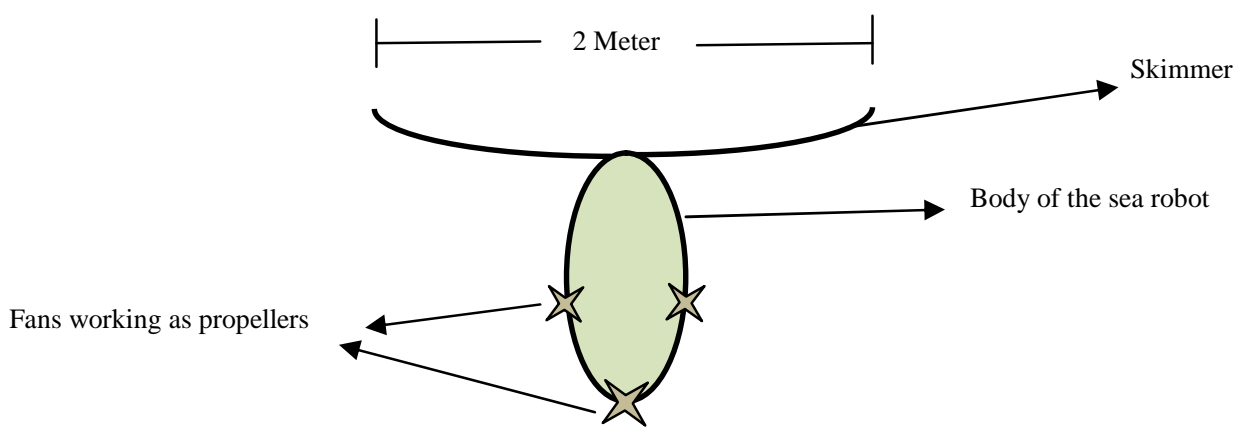

Figure 2. The Shape of the proposed robot 


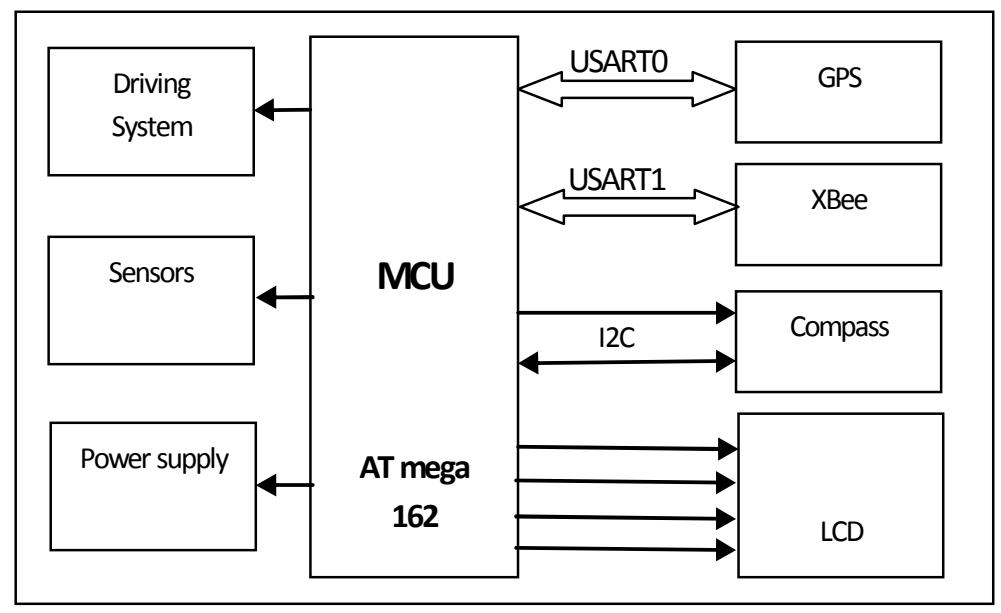

Figure 3. Robot main components connections

\section{RobOt DESIGN}

Figure 2. Robot main components connections

The robot was designed to operate on the surface of water (ship robot) equipped with a half oval shape skimmer with radius of 2 meter or boom (barriers that round up oil spill and lift the oil spill off the water) as shown in Figure 2 to push a floating oil spill to some position for better skimming and easier cleaning up. The design should be simple, low cost, easily maintained. Main components were enclosed in waterproof packaging and made in large numbers of it as it will be used as a swarm of robots on water. Also the system should meet the basic requirements for any swarm robotic system in mobility, sensing, localization and navigation. Each robot in the swarm was equipped with a GPS module, transceiver module, and a digital compass in addition to the main components on the main board such as micro-controller, voltage regulator, LCD, motor driver IC, etc. The expenses for all the mentioned components can go up to few hundred dollars for a single unit.

1. Chassis: It should be oval shaped to minimize resistance of the body with water, made of strong thick plastic.

2. A PCB board fixed inside the body with a microcontroller, GPS module, digital compass and one transceiver module for communication. This microcontroller had two USARTs one connected to the GPS and the other to transceiver module, the digital compass connected using two wire interface I2C as shown in Figure 3. Along with an LCD, motor driver IC, switches placed on the board to show status during testing.

3. Power supply: As the size of the robot was relatively big, there was enough place for an additional battery pack for backup, both the batteries were rechargeable from a solar panel fixed on the top of the robot. A solar engine with a switching process can change charging current from the main battery to the backup battery when the main battery voltage reaches a threshold value and vice versa.

4. Motors control: Mathew [17] talked about underwater robotic system by using vertical and horizontal thrusters to move the underwater robot in three dimensions. The current robot is a ship robot that can move in two dimensions so the same idea can be applied to drive the robot horizontally by using the horizontal thrusters to allow the robot to go forward, backward and turn. Each thruster had a motor that controls the thrusters using Pulse Width Modulation (PWM). 
5. Sensing System: usually robots use more than one sensor to learn about the environment, but in this robot only one ultrasonic sensor was used to detect obstacles in front of robot as the obstacles in sea would be less than on the ground. This sensor is needed when robot operates in autonomous mode to detect other robots and vessels in the work space and avoid collisions with them.

6. Communication System: Communication between a robot and a base station or other robots is essential in applications involving a swarm of robots and each robot should know about other robots around it. This robot used transceiver module with wired antenna as a communication unit to send and receive data. The transceiver module is small in size, light weight, low power consumption and relatively cheap with its range going up to $1400 \mathrm{~m}$ and data rate up to $250 \mathrm{kbps}$. The transceiver module can be used to build a wireless mesh network between the robots and a base station.

Mesh networking is used in applications where the range between two points may be beyond the range of the two radios located at those points, but intermediate radios are in place that could forward on any messages to and from the desired radios. The protocol thus designed can handle a number of different radios automatically to form a network without user intervention. The protocol within the radios takes care of retries, acknowledgements and data message routing. The primary advantage of the designed protocol lies in its ability to offer low power and extended battery life. This type of communication helped in controlling and monitoring each robot in the working space and provided more control on the robots to the coordinator when working in manual control

7. Skimmer: consists of two flat bars each of one meter length, $1-3 \mathrm{~cm}$ width and $15-25 \mathrm{~cm}$ height made of light material such as plastic. It is used by the robot to push and collect oil spill to certain positions. It is fixed at the front of robot, and design to be easily folded when moving backward as shown in Figure 4 for faster moving towards the work space and when returning back to the vessel after finishing the job.

8. Monitoring: the status of any robot in the swarm can be monitored as these robots keep on sending exchange message periodically to the base station they are moving. Written in any high level language such as C\#, the software can save robots' data (heading and coordinates) and use it for monitoring or even tracking with other robots as shown in Figure 5. However, the boundary of the working area represented by the rectangle ABCD. 


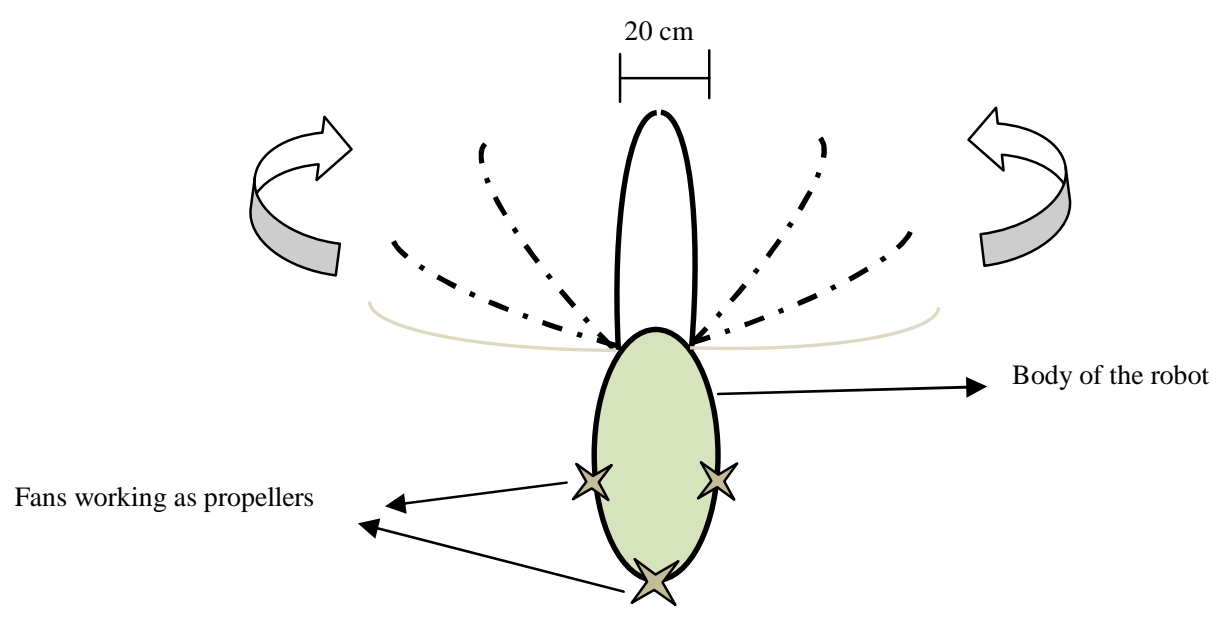

Figure 4 The Shape of the proposed robot with folded

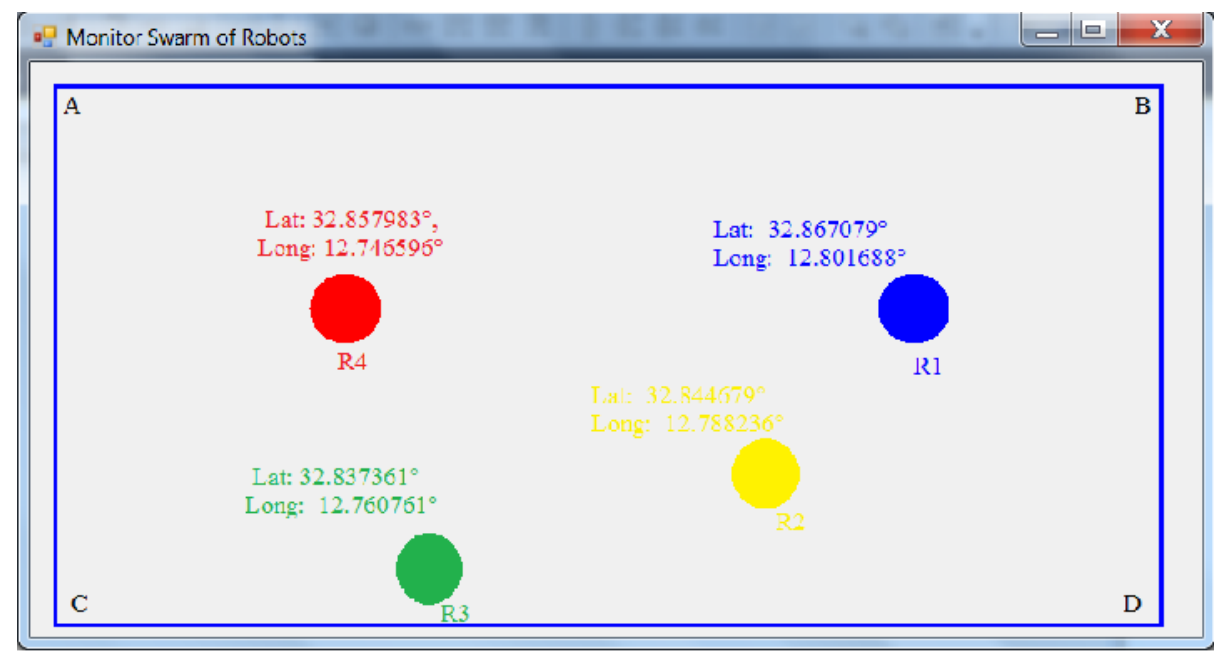

Fig 5. Program Monitoring a Swarm of Robots

\section{NAVIGATION OF THE SWARM}

Robots used on sea water can navigate easier than mobile robots on the ground, however the obstacles in sea are very rare and that enables ship robots to maneuver and move easily and reaching destination fast. Publicly available software such as Google Earth would be of not much help in operations in sea because there are no landmarks in the sea. The GPS module provides a packet of data contains latitude, longitude, altitude, date, time etc. The dataset can be used in different ways to be used by a dispatching algorithm and calculate distance, midpoint, heading etc.

The robot knows about its direction (heading) from the north by the digital compass onboard, and when the angle between the two GPS points $(\alpha)$ is calculated the robot can rotate in the same place by the value of $\alpha$ as show in Figure 6 while moving towards the destination. Based on data from the GPS and the digital compass a dispatching algorithm was used to calculate angle and 
distance to the center of the spill and distribute the swarm of robot on the boundary of the working area by sending wirelessly (broadcast) a packet containing the GPS coordinates of the center of the oil spill. Microcontroller on each robot was programmed to read the GPS coordinates through the transceiver module and start communicating and negotiating with other robots to take a decision where to go on the boundary of the spill. Each individual in the swarm will stop on the boundary by a specific angle ( $\alpha \mathrm{i})$ and distance (di) to the center of the spill, the robot with smaller $\alpha$ would stop in North side with $0^{\circ}$ degree next will stop with the $\alpha$ i degree and so on. Finding the angle between two GPS points is based on following expression:

$$
\alpha=\tan ^{-1}(y 2-y 1 / x 2-x 1)
$$

where: y1: current latitude, y2: destination latitude

$\mathrm{x} 1$ : current longitude, $\mathrm{x} 2$ : destination longitude

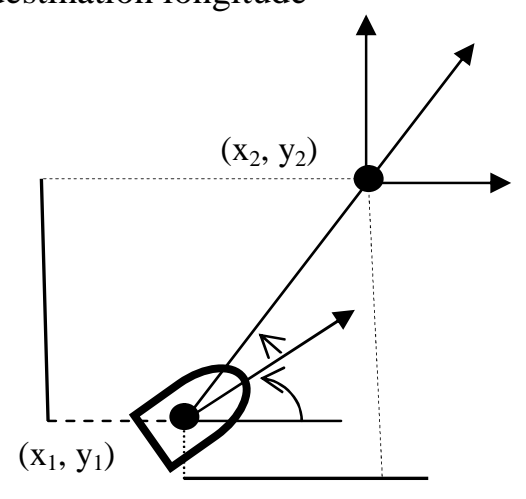

Figure 6. The angle between two GPS points

\section{Operation OF THE SWARM}

A coordinator determines the position and center of the oil spill first using a GPS receiver, then a barge carrying the robots move to the working place. According to the status of the oil spill (shape, area covered, one spill or many, direction and speed of wind, etc.) the coordinator can dispatch a swarm of robots to surround and collect the oil spill, then the barge with an oil sucking equipment move it for another place to collect/dispose the oil in a safer way.

Each robot works in two modes, viz., autonomous mode and remote control mode.

- Autonomous Mode: as the robot has a transceiver, GPS module and digital compass, a data packet is broadcasted using XBee module to all robots to move to target GPS coordinates. On the robot the microcontroller will read the received coordinates and make simple calculations to find the angle between the two GPS coordinates (current coordinates and destination coordinates). The microcontroller continues comparing the angle and instructing the robot to keep moving towards the destination. As it works in autonomous mode, an ultrasonic sensor and collision avoidance algorithm are used to avoid collision with other robots and barges in the work space. 
International Journal of Advanced Information Technology (IJAIT) Vol. 2, No.4, August 2012

- Remote Control Mode: The coordinator can take control to dispatch the swarm of robots in the working space, in remote control mode, the microcontroller converts the remote signals to commands that are executed by the robot control system.

\section{Issues in Oil Spill Cleaning UP}

There would be a number of aspects based on which the swarm of robots can be dispatched in water to collect the oil spill and prevent it from stretching and spread wider. Some of them to be handled for successful recovery from oil spills are as following:

1. The location of the oil spill (in the middle of sea or near a coastline).

2. Area covered with oil.

3. Thickness of the oil layer.

4. Spill in one large portion or spread across into many portions of varying sizes.

5. Sea state - night time, high-tide, etc. should be carefully examined and safety of the robots should be taken up on highest priority.

6. Skill of the operators

7. Number of vessels needed to accomplish the task

8. Storage capacity in vessels that carry the recovered oil for disposal.

9. Lot of time would be required to collect the spills together and hence battery based robots will not be helpful. Alternate methods such as using solar energy should be considered.

10. Efficiency of the algorithms and protocols for communication between the robots.

Case 1: Oil spill is nearer to coastline and the aim is to prevent the spill from reaching the coastline. The swarm of the sea robots equipped with skimmer or a boom can be distributed in straight line with fixed distances adjacent to each other to form a line and start moving opposite to the coast direction as shown in Figure 7.

Case 2: If the oil spill is in polygon shape, the center of the spill can be determined and the swarm can start the task, but in case the robots will work as a straight line or a curve to prevent the oil spill from reaching sea shore or a coastline, the dispatching algorithm is used in different ways by the coordinator. According to the number of robots in the swarm, it can be decided how much distance can be covered and end points of the line. Then distribute the robots in fixed distances on that line to prevent a spill from reaching particular area.

Further, if oil spill is connected and taking polygon shape inside the sea, the aim is to collect the oil spill or move it to another position for better and faster cleaning. The swarm of sea robots can be dispatched on the boundary of the spill to surround it up and wait for the vessels to take up the oil and discharge it in safer way. 
Case 3: Oil spill is one piece and the swarm of robots is dispatched to the boundary of the spill and try to be connected as much as possible using some sensors to ensure that there is no leakage between any two adjacent robots, the swarm can be in the same position to surround the oil spill (like in the night time) or can move the spill to any required position.

Case 4: There are many oil spills either inside the sea or near a coastal line. The aim is to prevent the spill from spreading wider. The coordinator or master will determine the GPS coordinates of the center of each spill and will start using task partitioning algorithm by dividing the swarm of sea robots into teams and then a dispatching algorithm is used to enable each sub team to collect sub-spill as shown in Figure 8. The outcome will be either surrounding the spill or move it to another position.

\section{Conclusion}

A swarm of sea robots can be used in collecting an oil spill in the sea more successfully and efficiently than most of the other methods and save the environment from a real threat. This way it will be possible to reduce human intervention and enhance accuracy even in the most hostile environmental conditions. Using swarm of sea robots instead of using barges specially in collecting small oil spills will save money and time.

As future extension of work, design of chassis of the robot with most of the components considered for integration on a single PCB, dispatching algorithm and partition algorithm could be implemented.

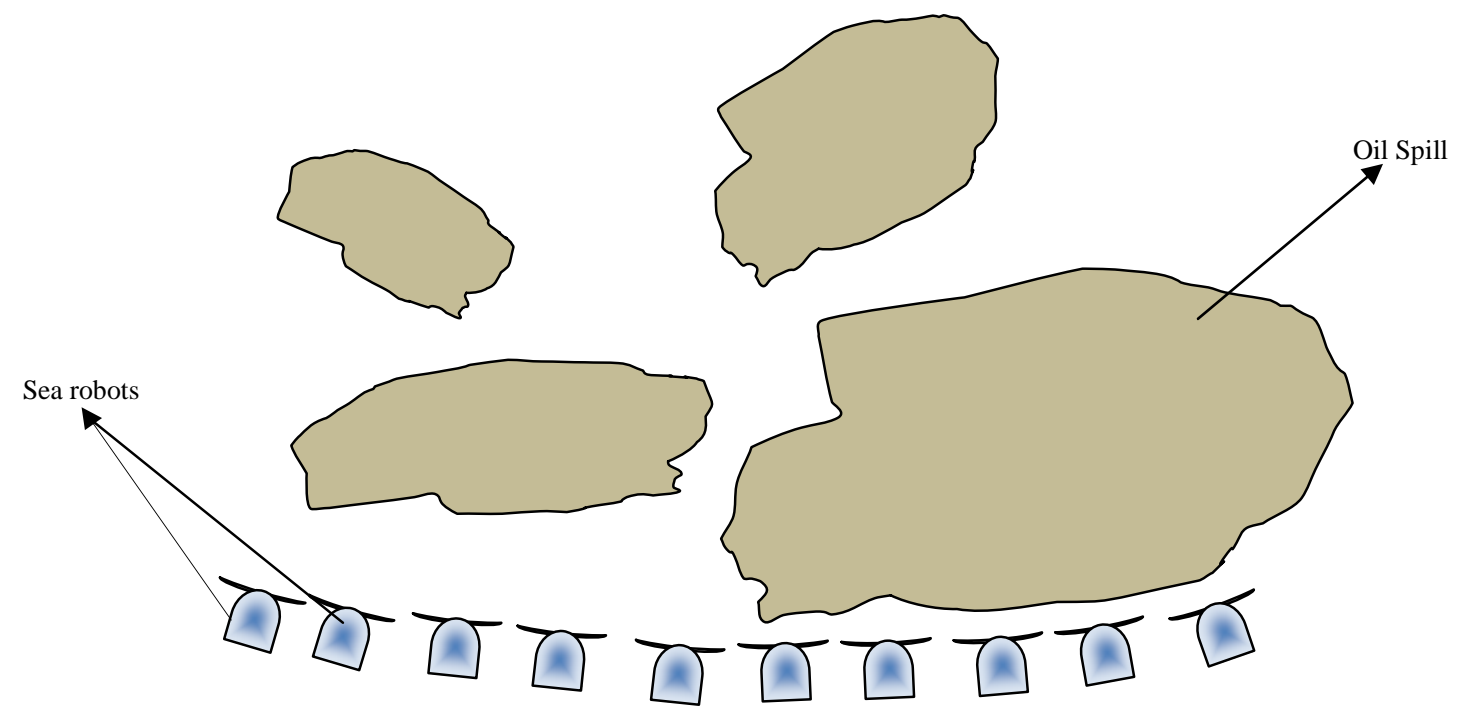

Figure 7 A swarm of sea robots arranged in straight line to move oil spills away from coast line 


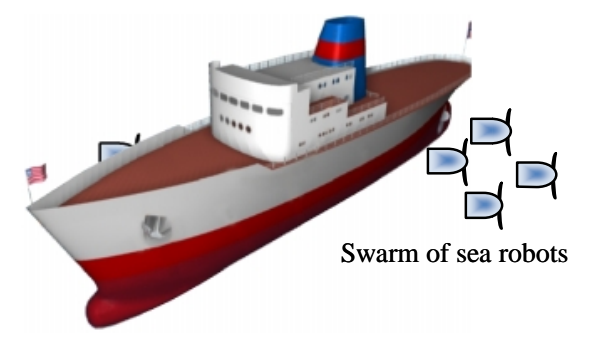

Barge carry the sea robots

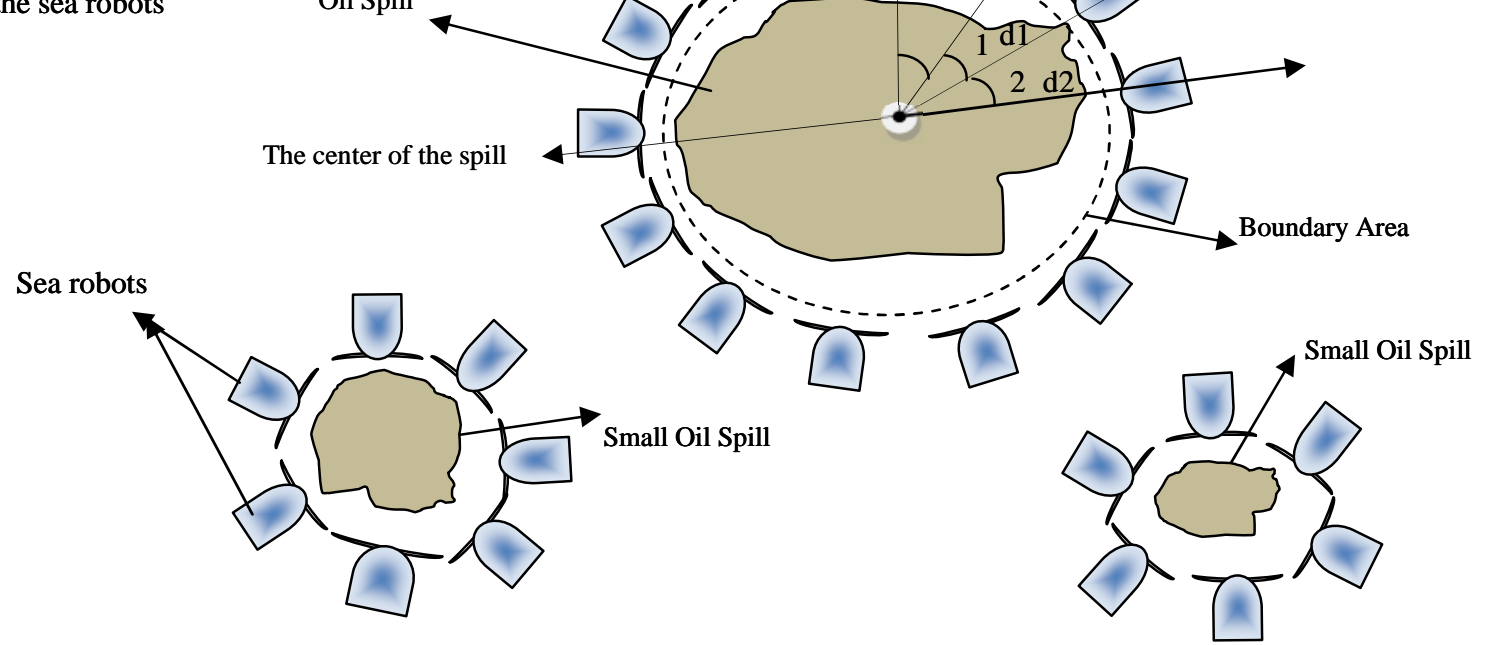

Figure 8. Dispatch swarm of sea robots around oil spills

\section{REFERENCES}

[1] Zhao Qingling and Li Ying, "Monitoring Marine Oil-spill Using Microwave Remote Sensing Technology" Electronic Measurement and Instruments, International Conference, pp 4-69, 4-72, 2007.

[2] Chase, C.R. Van Bibber, S. Muniz, T.P, "Development of a non contact oil spill detection system”, Ocean proceeding of MTS/IEEE, Vol. 2, pp 1352-1357, 2005.

[3] Brekke, C.; Solberg, A. (2005). Oil spill detection by satellite remote sensing. Remote Sensing of Environment 2005, 95, $1-13$.

[4] Brown, C.; Fingas, M. Development of airborne oil thickness measurements. Marine Pollution Bulletin 47, 485-492, 2003 b.

[5] Øistein Johansen, Henrik Rye, Cortis Cooper. "Deep spill-field study of a simulated oil and gas blowout in deep water," Spill Science \& Technology Bulletin, vol. 8, no. 5-6, pp. 433-443, 2003.

[6] Song-ping Zhu, Dmitry Strunin. "A numerical model for the confinement of oil spill with floating booms," Spill Science \&Technology Bulletin, vol. 7, no. 5-6, pp. 249-255, 2002.

[7] Ming Xiao, Qingjun Gao, Jianguo Lin, Wei Li, Xiao Liang. "Simulation of submarine pipeline oil spill based on wave motion " 2nd conference on computer modeling and simulation, 2010

[8] S.D. Wanga, Y.M. Shena, Y.H. Zheng. "Two-dimensional numerical simulation for transport and fate of oil spills in seas," Ocean Engineering, vol. 32, pp. 1556-1571, 2005.

[9] Wikipedia available at http://en.wikipedia.org/wiki/Oil_spill”,

[10] Mazmdouh T. Ghannam and Omar Chaalal, "Oil spill cleanup using vacuum technique ," Fuel, vol. 82, pp. 789-797, May 2003.

[11] N. E. Leonard and J. G. Graver, "Model-based feedback control of autonomous underwater gliders," IEEE Journal of Oceanic Engineering, vol. 26, no. 4, pp. 633-645, 2001. 
International Journal of Advanced Information Technology (IJAIT) Vol. 2, No.4, August 2012

[12] P. R. Bandyopadhyay, "Trends in biorobotic autonomous undersea vehicles," IEEE Journal of Oceanic Engineering, vol. 30, no. 1, pp. 109-139, 2005

[13] T. Fukuda, A. Kawamoto, F. Arai, and H. Matsuura, "Steering mechanism of underwater micro mobile robot," in Proceedings of the IEEE Conference on Robotics and Automation, 1995, pp. 363368.

[14] P. E. Hagen, N. Storkersen, K. Vestgard, and P. Kartvedt, "The HUGIN 1000 autonomous underwater vehicle for military applications," OCEANS 2003. Proceedings, vol. 2 pp. $1141-1145,2003$.

[15] Xiaobo Tan, Kim, D. Usher, N. Laboy, D. Jackson, J; Kapetanovic, A. Rapai, J. Sabadus, B. Xin Zhou, "An Autonomous Robotic Fish for Mobile Sensing”, Intelligent Robots and Systems, pp 54245429, 2006.

[16] Pascoal, A., Silvestre, C., Oliveira, P. (2006) Advances in unmanned marine vehicles. IEE Control Series, ch. Vehicle and mission control of single and multiple autonomous marine robots, pp. 353 386.

[17] Joordens, M.A." Design of a low cost underwater robotic research platform", System of Systems Engineering, SoSE IEEE International Conference, pp 1-6, 2008.

\section{Authors}

Emaad Mohamed H. Zahugi received his bachelor's degree in Computer Science from Tripoli University, Libya. He received his master's degree in Computer Science \& Engg. from Jamia Hamdard, New Delhi, India. He is currently a $\mathrm{PhD}$ scholar at Lingaya's University, Faridabad, India. He is working in the area of swarm intelligence and robotics. He has 6 papers to his credit. He is a member of IEEE His areas of interests include robotics, swarm intelligence and wireless communications.

Mohamed Mustafa AB Shanta received his bachelor's degree in Computer Science from Wojskowa Akademia Techniczna, Poland. He received his master's degree in Computer Applications from Jamia Hamdard, New Delhi, India and is currently a PhD scholar at Lingaya's University, Faridabad, India. He is working in the area of image processing. His areas of interests include image processing and wireless communications.

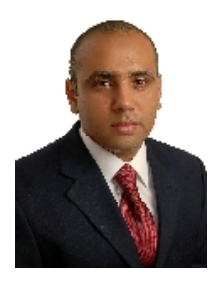

Dr. T. V. Prasad received his bachelor's and master's degree in Computer Science from Nagarjuna University, AP India. He received PhD degree from Jamia Millia Islamia University, New Delhi, India. He has over six years teaching at UG and PG levels, and has also worked as Deputy Director for 11 years in the Bureau of Indian Standards, New Delhi. He was with Lingaya's University, Faridabad, Haryana, India for five years as Professor \& Head, Dept. of Computer Science \& Engineering, Dean of R\&D and

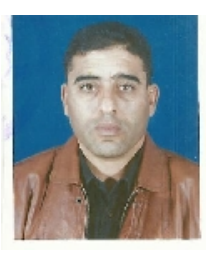
Industrial Consultancy and Dean of Academic Affairs. Currently, he is associated with the Visvodaya Technical Academy, Kavali, Andhra Pradesh as Dean of Computing Sciences. He is also a trained auditor for Information Security Management Systems (ISMS) as per ISO 27001 standard. He has over 72 papers and six books to his credit. He is a member of IEEE, IAEng, CSI, and Life member of APBioNet and ISRS. His areas of interests include artificial intelligence, bioinformatics, consciousness studies, computer organization and architecture. 